\title{
Anal mucosectomy for haemorrhoids: should we start to speak Chinese?
}

\section{Dindo* and D. Hahnlosert}

*Department for Visceral, Thoracic and Vascular Surgery, City Hospital Triemli, Zurich, Switzerland and †Department of Visceral Surgery, University Hospital CHUV, Lausanne, Switzerland

Received 6 April 20 I2; accepted II September 20 I2; Accepted Article online II February 2013

\begin{abstract}
Aim Circular stapled mucosectomy is the standard therapy for the treatment of symptomatic third-degree haemorrhoids and mucosal prolapse. Recently, new staplers made in China have entered the market offering an alternative to the PPH stapling devices. The aim of this prospective randomized study was to compare the safety and efficacy of these new devices.

Methods Fifty patients with symptomatic third-degree haemorrhoids were randomized to mucosectomy either by using stapler A (CPH32; Frankenman International Ltd, Hong Kong, China; $n=25$ ) or stapler B (PPH03; Ethicon Endo-Surgery, Spreitenbach, Switzerland; $n=25$ ). All procedures were performed by two experienced surgeons. After the stapler was fired by one surgeon, the other surgeon, who was blinded for stapler type, evaluated the stapler line. Postoperative outcome including pain, complications and patient satisfaction were analysed.
\end{abstract}

Results Demographic and clinical features were no different between the groups. There was no significant difference regarding venous bleeding $(P=0.55)$, but arterial bleeding was significantly more frequent when stapler B was used $(P<0.001)$. This led to significantly more suture ligations $(P=0.002)$. However, no differences regarding operation time $(P=0.99)$, weight of the resected mucosa $(P=0.81)$ and height of the stapler line (anterior, $P=0.18$; posterior, $P=0.65$ ) were detected. Postoperative pain scores (visual analogue scale) and patient satisfaction were no different either $(P=0.91$ and $P=0.78$, respectively). No recurrence or incontinence occurred during follow-up.

Conclusions $\mathrm{CPH} 32$ required significantly fewer sutures for bleeding control along the stapler line after circular mucosectomy. However, operation time, rate of postoperative complications and patient satisfaction were similar in both groups.

Keywords Haemorrhoidectomy, Longo

\section{What is new in this paper?}

This prospective randomized trial demonstrates advantages regarding the need for sutures for bleeding control along the stapler line after circular mucosectomy for haemorrhoids using a new stapling device.

\section{Introduction}

Stapled mucosectomy was first described in 1998 for the treatment of symptomatic haemorrhoids [1]. This new surgical concept corrects mucosal prolapse and haemorrhoidal disease by means of the circumferential excision of the supra-haemorrhoidal rectal mucosa. The operation involves the lifting and fastening of the anal mucosa and of the haemorrhoids in their natural anatomical site. The results of the tens of thousands of

Correspondence to: Dieter Hahnloser, MD, Department of Visceral Surgery, University Hospital CHUV, Rue du Bugnon 46, CH-IOII Lausanne, Switzerland.

E-mail: dieter.hahnloser@chuv.ch operations performed so far confirmed that this method is safe and effective. There is also good evidence that this technique offers advantages in terms of less postoperative pain, shorter hospital stay and time off work as compared with conventional haemorrhoidectomy [2-5].

The most frequently occurring early complication of this procedure is staple dehiscence and bleeding. Partial staple dehiscence may be observed in $1 \%$ of cases and the patients need additional sutures [6]. Bleeding complications requiring surgical reintervention may occur in up to $2-4 \%$ of patients $[6,7]$. For most of the studies, the PPH03 circular stapler was used (Ethicon EndoSurgery, Spreitenbach, Switzerland). Recently, several competitors, mainly Chinese, have entered into the 
market thus offering a greater variety of stapling devices for use. However, there are few data yet published regarding the safety and efficacy of these new devices. The aim of this study was to evaluate the safety and efficacy of the CPH32 stapler (Frankenman International Ltd, Hong Kong, China) in a prospective randomized trial.

\section{Method}

All consecutive patients who were scheduled for stapled mucosectomy for third-degree haemorrhoids during a 9-month period at the University Hospital Zurich were included in this study. The primary end-point was intraoperative bleeding and sutures used for haemostasis at the stapled line. Secondary end-points were pain on the day of surgery and patient satisfaction 3 weeks after surgery. After having obtained informed consent, 50 patients with symptomatic third-degree haemorrhoids were randomized to mucosectomy either by using stapler A (CPH $32 ; n=25)$ or stapler B (PPH03; $n=25)$. Randomization was performed using sealed envelopes, which were only opened in the operation room.

The procedure was performed as previously published [1]. All operations were performed under general or spinal anaesthesia and in an outpatient setting. All procedures were performed by two experienced surgeons $(\mathrm{DD}, \mathrm{DH})$ in a standardized manner. The pursestring suture was placed at the base of the haemorrhoidal cushions. After the stapler was fired by one surgeon, the other surgeon, who was blinded for stapler type, was called into the operation room to evaluate the quality of the stapler line. Any devices which could have indicated which stapler was used were removed from the operating room. The stapler line was assessed in a standardized manner regarding bleeding (arterial, venous), need for additional stitches (determined by the blinded surgeon), height of stapler line (anterior and posterior) and stapler line dehiscence. Dehiscence was defined as mucosal tear at the staple line. The height of the staple line was anteriorly and posteriorly measured from the anocutaneous junction to the staple line and the weight of the resected specimen was measured (Fig. 1).

Postoperative pain was assessed on the day of surgery at discharge using a visual analogue scale (VAS; 0-10). All patients were seen again in the outpatient clinic at 1 month. Patient satisfaction was assessed during this follow-up visit (using a VAS). Postoperative complications were recorded as 30-day morbidity. All procedures were performed as 1-day surgery. The study was approved by the local ethical committee of the Univer- sity Hospital Zurich. No financial support for this study was received from either company. The authors have no conflict of interest.

\section{Statistical analyses}

Data are expressed as mean (SD) unless otherwise stated. Sample size was calculated before the study: Power calculation revealed a sample size of 25 patients in each group needed to detect a $20 \%$ difference in stitches needed $(80 \%$ power at a $5 \%$ significance level). Qualitative variables were analysed using a $\chi^{2}$ Pearson test and Fisher's exact test. The Mann-Whitney $U$-test was used for continuous variables. $P<0.05$ was considered statistically significant.

\section{Results}

Fifty-three operations were performed during the study period. One patient declined to participate and for two patients one of the two surgeons was not available. Fifty patients were randomized. Demographic and clinical features were no different between the groups (Table 1). Operation time was $30 \mathrm{~min}$ with no difference between the groups $(P=0.94)$. While no significant difference regarding venous bleeding was noted $(P=0.55)$, arterial bleeding was significantly more frequent when stapler B was used $(P<0.001)$. This led to significantly more suture ligations (mean 4 vs 2 sutures per patient; $P=0.002)$. However, no differences regarding operation time, weight of the resected mucosa $(P=0.81)$ and the height of the stapler line (anterior, $P=0.18$; posterior, $P=0.65$ ) were detected (Table 2). All procedures in this study were done as 1-day surgery. There were no readmissions during the follow-up. Postoperative pain scores (VAS) were no different either $(P=0.91)$. In one patient, in whom stapler B was used, a postoperative anal fissure had to be treated surgically. No other complications occurred within 30 days. No recurrence or incontinence was found during follow up. At 30 days, patient satisfaction was equal for both stapler types $(P=0.78)$.

\section{Discussion}

Many studies have confirmed the advantages of stapled anal mucosectomy for the treatment of symptomatic haemorrhoids compared with conventional surgical excision in regard to perioperative pain and patient comfort $[8,9]$. However, postoperative bleeding occurring in between 1.8 and $4 \%$ of cases $[6,7,10]$ and higher recurrence rates [8] have been a major concern. Initially, the PPH0l stapler (Ethicon Endo-Surgery, Spreitenbach, 


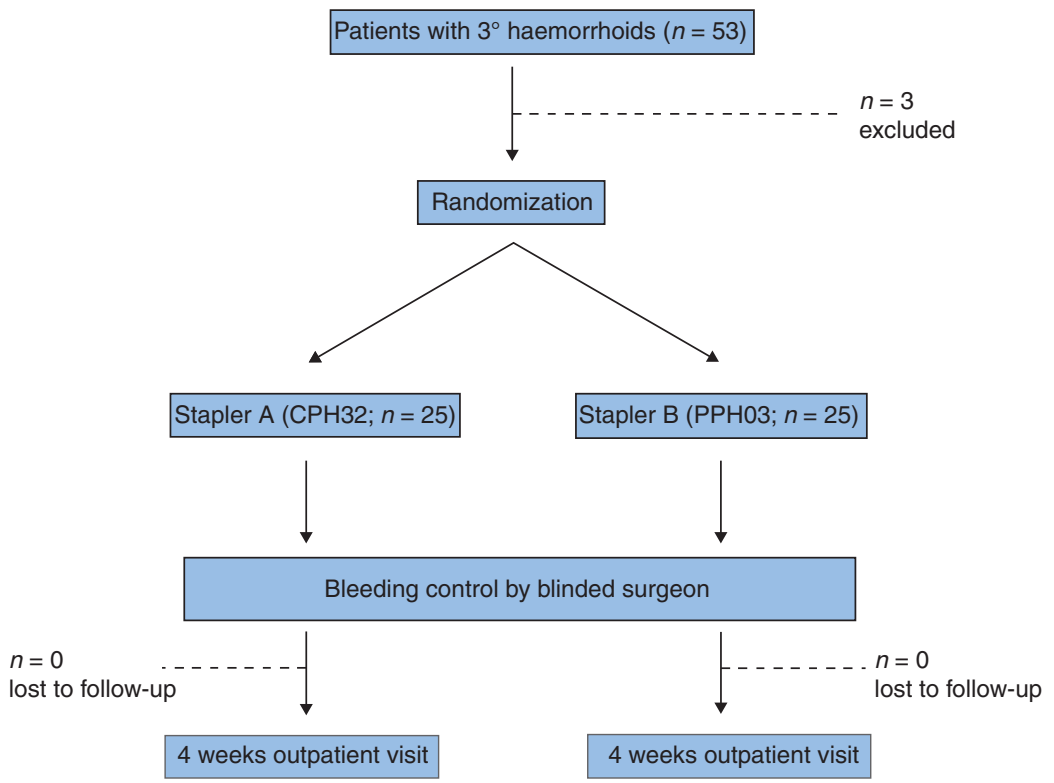

Figure I Flow chart.

Table I Clinical outcome parameters.

\begin{tabular}{lccc}
\hline & Stapler A & Stapler B & $P$-value \\
\hline $\begin{array}{l}\text { Anaesthesia type }(n) \\
\quad \text { General }\end{array}$ & 17 & 16 & 0.95 \\
$\begin{array}{l}\text { Spinal } \\
\begin{array}{l}\text { Operative time } \\
\text { (min; range) }\end{array}\end{array}$ & $80(15-70)$ & $30(15-60)$ & 0.94 \\
$\begin{array}{l}\text { Pain score at } \\
\text { discharge (VAS) }\end{array}$ & $2(0-6)$ & $2(0-5)$ & 0.91 \\
One-day surgery $(n)$ & 25 & 25 & 1 \\
\hline
\end{tabular}

VAS, visual analogue scale.

Table 2 Intra-operative parameters.

\begin{tabular}{lccr}
\hline & Stapler A & Stapler B & $P$-value \\
\hline Height of stapled line & & & \\
anterior $(\mathrm{mm})$ & $1.5(0.8-1.5)$ & $1.5(1.0-2.2)$ & 0.18 \\
posterior $(\mathrm{mm})$ & $1.5(0.5-1.8)$ & $1.2(1.0-1.8)$ & 0.65 \\
Weight of resected & $5(3-7)$ & $5(3-7)$ & 0.81 \\
specimen $(\mathrm{g})$ & & & \\
Venous bleeding $(n)$ & $2(0-3)$ & $1(0-4)$ & 0.55 \\
Arterial bleeding $(n)$ & $1(0-4)$ & $0(0-1)$ & $<0.001$ \\
Sutures needed $(n)$ & $4(0-11)$ & $2(0-3)$ & 0.002 \\
\hline
\end{tabular}

Switzerland) was used in many studies. Using this stapling device, intra-operative bleeding requiring additional sutures has been reported to occur in $50-60 \%$ of patients [11-14]. Bleeding has been shown to be lower using the PPH03 instead of the PPH0l staper [14].

Additional sutures were necessary in $50 \%(15 / 30)$ in the PPH01 group compared with only $11 \%(4 / 30)$ in the PPH03 group. Moreover, the rate of tenesmus or discomfort during defaecation was higher in the PPHOl group. As a consequence, the PPH03 stapler has become the standard device for stapled mucosectomy in recent years.

Bleeding along the staple line is still frequently observed. In a study reporting on 695 patients treated with the PPH03, 49.8\% of patients required additional sutures to control bleeding at the staple line [10]. Therefore, additional improvement in bleeding control would be desirable. Our prospective randomized study reveals advantages of the $\mathrm{CPH} 32$ over the PPH03 in terms of haemostasis. However, operation time, postoperative complications, short-term outcome and patient satisfaction were similar. In our study, we did not observe a correlation between haemostatic sutures and postoperative pain as in the study of Arroyo et al. [14]. Pain scores and patient satisfaction were not statistically different between the CPH32 and the PPH03. The better haemostasis of the CPH32 stapler might be explained by the number and configuration of the staplers. The additional number of staples as provided in the CPH32 (32 vs $28 \mathrm{~mm}$ ) may explain this difference. However, the better haemostasis along the staple line did not translate into a faster operation time or fewer complications in our study as in the study of Arroyo et al. 
A possible limitation of this study is the focus only on short-term outcomes and the lack of long-term followup. Despite good initial results, long-term follow-up is required to detect recurrences. Therefore, we cannot draw conclusions about the long-term efficacy of the CPH32. Nevertheless, intra-operative bleeding control is superior using the $\mathrm{CPH} 32$ compared with the PPH03. However, because there is no difference in clinically relevant outcome parameters between the CPH32 and the PPH03, both stapling devices are equally safe and effective.

\section{Authors' contributions}

DD and DH have contributed equally to the concept, design, acquisition of data, analysis and writing of the manuscript.

\section{Conflict of interest}

None.

\section{References}

1 Longo A. (1998) Treatment of hemorroidal disease by reduction of mucosa and hemorrhoidal prolapsed with a circular suturing device: a new procedure. Proceedings of the 6th World Congress of Endoscopic Surgery, June 3-6, 1998, Rome, Italy. Bologna: Monduzzi; 777-84.

2 Boccasanta P, Capretti PG, Venturi M et al. Randomised controlled trial between stapled circumferential mucosectomy and conventional circular hemorrhoidectomy in advanced hemorrhoids with external mucosal prolapse. Am J Surg 2001; 182: 64-8.

3 Hetzer FH, Demartines N, Handschin AE, Clavien PA. Stapled vs excision hemorrhoidectomy: long-term results of a prospective randomized trial. Arch Surg 2002; 137: 337-40.

4 Cheetham MJ, Cohen CR, Kamm MA, Phillips RK. A randomized, controlled trial of diathermy hemorrhoidectomy vs.stapled hemorrhoidectomy in an intended day-care setting with longer-term follow-up. Dis Colon Rectum 2003; 46: 491-7.

5 Ganio E, Altomare DF, Gabrielli F, Milito G, Canuti S. Prospective randomized multicentre trial comparing stapled with open haemorrhoidectomy. Br J Surg 2001; 88: 669-74.

6 Ommer A, Hinrichs J, Möllenberg H, Marla B, Walz MK. Long-term results after stapled hemorrhoidopexy: a prospective study with a 6-year follow-up. Dis Colon Rectum 2011; 54: 601-8.

7 Oughriss M, Yver R, Faucheron JL. Complications of stapled hemorrhoidectomy : a French multicentric study. Gastroenterol Clin Biol 2005; 29: 429-33.

8 Shao WJ, Li GC, Zhang ZH, Yang BL, Sun GD, Chen YQ. Systematic review and meta-analysis of randomized controlled trials comparing stapled haemorrhoidopexy with conventional haemorrhoidectomy. Br J Surg 2008; 95: 147-60.

9 Jayaraman S, Colquhoun PH, Malthaner RA. Stapled versus conventional surgery for hemorrhoids. Cochrane Database Syst Rev 2006 Oct. 18; (4).

10 Knight JS, Senapati A, Lamparelli MJ. National UK audit of procedure for prolapsing haemorrhoids on behalf of the Association of Coloproctology of Great Britain and Ireland. Colorectal Dis 2008; 10: 440-5.

11 Mehigan BJ, Monson JR, Hartley JE. Stapling procedure for haemorrhoids versus Milligan-Morgan haemorrhoidectomy: randomised controlled trial. Lancet 2000; 355: 782 85.

12 Lehur PA, Gravie JF, Meurette G. Circular stapled anopexy for haemorrhoidal disease: results. Colorectal Dis 2001; 3: 347-79.

13 Smyth EF, Baker RP, Wilken BJ, Hartley JE, White TJ, Monson JRL. Stapled versus excision haemorrhoidectomy: long term follow-up of a randomized controlled trial. Lancet 2003; 361: 1437-8.

14 Arroyo A, Pérez-Vicente F, Miranda E et al. Prospective randomized clinical trial comparing two different circular staplers for mucosectomy in the treatment of hemorrhoids. World J Surg 2006; 30: 1305-10. 